\title{
The Theory of a Stable High-Speed Externally Pressurized Gas-Lubricated Bearing
}

\author{
H. Sixsmith and W. A. Wilson
}

(January 15, 1964)

\begin{abstract}
Externally pressurized gas-lubricated bearings have been used successfully to support the shaft of a miniature, high-speed expansion turbine. The bearings incorporate stabilizing cavities to suppress whirl at high speeds. The theory of the bearing design is presented in detail.
\end{abstract}

\section{Introduction}

In the field of gas liquefaction and low temperature refrigeration, small high-speed expansion turbines are often employed as the source of refrigeration. In the smaller sizes of machines however, the life and speed of conventional bearings are limited, and consequently a lower limit is set to the practical size of such machines. This limitation may be alleviated by the use of gas-lubricated bearings to support the shaft. The bearings may be lubricated with process gas, thus eliminating the risk of contamination with oil. Since there is no solid friction, there should be no wear and consequently the life of the bearings should be of indefinite duration.

In the operation of a gas-lubricated bearing, the surface of the shaft must never touch the surface of the bearing, since at the high-surface speeds usually employed, such contact may result in seizure and the consequent destruction of the bearing, Under normal operating conditions the shaft should be supported by forces due to pressure in the gas film between the shaft and the bearing and these forces must be such that the shaft is maintained in a position of stable equilibrium.

In certain types of gas-lubricated bearings, a form of instability known as whirl is sometimes observed, particularly at high speeds of operation. If the speed of the shaft is increased above a critical value, the center of the shaft orbits about the center of the bearing at a speed approximately one-half the speed of the shaft - commonly known as half-speed whirl.

Whirl may be stable or unstable: Stable whirl is characterized by the center of the shaft moving in a closed orbit about its former position of equilibrium. This is illustrated in figure 1. Unstable whirl (fig. 2 ) is characterized by the center of the shaft moving along a spiral orbit of increasing amplitude. The extent of the increase in amplitude is, of course, limited to the extent of the radial clearance between the shaft and the bearing.
The bearing described below has been specially designed to ensure stability even at the highest speeds of operation with helium gas as the pressurizing medium. The theory of this bearing was presented at the First International Symposium on Gas Lubricated Bearings [Sixsmith, 1959b]. In the present paper, the theory has been extended and applied to the design of the bearings of a helium-expansion turbine [Birmingham, Sixsmith, Wilson, 1962]. The

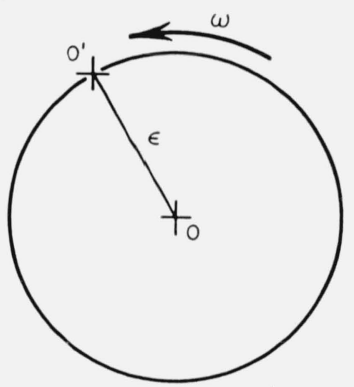

Figure 1. Whirl: constant amplitude.

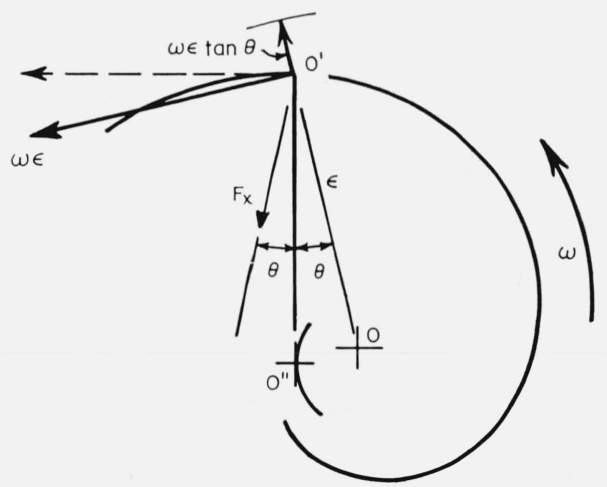

Figure 2. Whirl: increasing amplitude. 
theoretical approach used in this particular design and construction is outlined in the following detailed analysis.

\section{Shaft Whirl}

In the diagram shown in figure 2 , it is assumed that the shaft is vertical so that the only forces acting on the shaft are those due to pressure in the surrounding gas. At a given instant of time, the centers of the bearing and the shaft are situated at $O$ and $O^{\prime}$. The radial displacement $O O^{\prime}$ is represented by $\epsilon$. The shaft is moving to the left along' a spiral orbit. The resultant of all the forces due to gas pressure is represented by the force vector $\mathbf{F}_{\mathbf{X}}$. The instant center of the orbit of $O^{\prime}$ is situated at $O^{\prime \prime}$. The line $O^{\prime} O^{\prime \prime}$ bisects the angle between $\mathbf{F}_{\mathbf{x}}$ and the radius $O^{\prime} O$.

It will be assumed that, over a restricted range, the force $\mathbf{F}_{\mathbf{X}}$ varies directly as the radius $O O^{\prime}$, and, hence, behaves like a spring. The force $\mathbf{F}_{\mathbf{x}}$ may be resolved into three components.

$$
\mathbf{F}_{\mathbf{X}} \cos ^{2} \theta=Y\left(O O^{\prime}\right)
$$

which pulls the shaft toward the bearing center,

$$
\mathbf{F}_{\mathbf{X}} \sin \theta
$$

which accelerates the shaft center along the orbit, and a component

$$
\mathbf{F}_{\mathbf{X}} \cos \theta \sin \theta
$$

which provides the energy required to stretch the "spring".

The angular velocity $\omega$ of $O^{\prime}$ about $O$ is given by the equation

$$
m \omega^{2}\left(O O^{\prime}\right)=Y\left(O O^{\prime}\right)=Y \epsilon
$$

or

$$
\omega=\sqrt{\frac{Y}{m}}
$$

where $m$ is the effective mass of the shaft in the plane of the bearing.

The linear velocity $\omega\left(O^{\prime \prime} O^{\prime}\right)$ of the center of the shaft may be resolved into a tangential component

$$
\omega\left(O^{\prime \prime} O^{\prime}\right) \cos \theta=\omega \epsilon
$$

and a radial component

$$
\omega\left(O^{\prime \prime} O^{\prime}\right) \sin \theta=\omega \epsilon \tan \theta .
$$

Thus the line of centers may be regarded as rotating about the center $O$ with an angular velocity $\omega$, while the radial distance $\epsilon$ expands as it rotates. The rate of expansion is given by the equation

$$
\epsilon=\epsilon_{0} e^{\omega t \tan \theta}
$$

where $\epsilon_{0}$ is the value of the eccentricity when $t=0$. Thus if the direction of the resultant force lies ahead

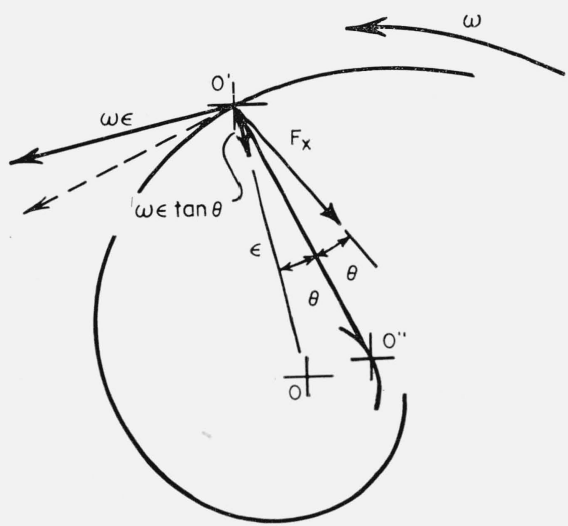

Figure 3. Whirl: decreasing amplitude.

of the line of centers ( $\theta$ positive), the diagram expands exponentially with time; conversely if the direction of the resultant force lies behind the line of centers ( $\theta$ negative) as shown in figure 3 , the diagram shrinks in toward the origin and the bearing should be stable, even though the force $\mathbf{F}_{\mathbf{X}}$ may be quite small.

In practice, the forces due to the film of gas may be far from linear, and at a certain speed of the shaft, if the eccentricity is increased from zero, the sign of the angle $\theta$ may change from positive to negative at some critical value of the eccentricity. Under these conditions the diagram should expand asymptotically toward the critical value of the eccentricity and whirling of stable amplitude should occur. This is often observed in practice.

\section{Fluent Film Lubrication}

The action of a simple sleeve bearing is illustrated in figure 4. A shaft of radius $r$ is surrounded by a sleeve of radius $r+\delta$, the interspace being filled with

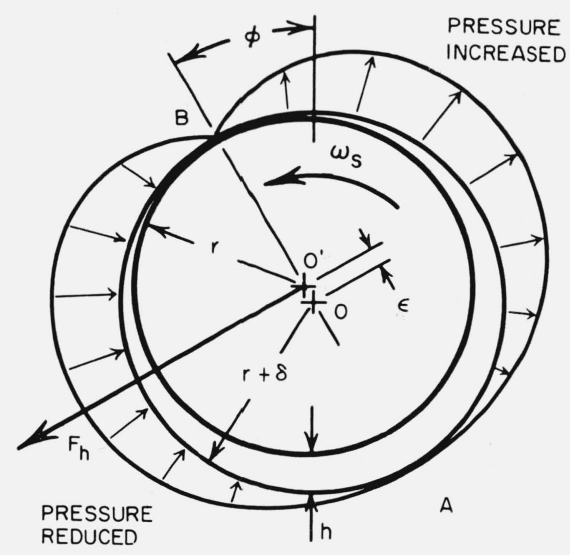

Figure 4. Hydrodynamic pressure distribution. 
a continuous film of lubricant. The center of the shaft $O^{\prime}$ is displaced from the center $O$ of the sleeve by a small distance $\epsilon$. If the shaft rotates counterclockwise, the film of lubricant is carried into a converging channel on the upper right-hand side of the diagram, and out of a diverging channel on the lower left-hand side of the diagram. Thus the pressure is increased on the upper right-hand side and reduced on the lower left-hand side. The resultant force due to the pressure distribution is at right angles to the eccentricity. This is true, however, only for very small values of the eccentricity where compressibility effects can be neglected; at larger values of the eccentricity, compressibility effects introduce an asymmetry and a component of force directed toward the center of the bearing is generated.

Suppose the eccentric displacement of the shaft from the center of the bearing is maintained at a fixed value by means of a short link free to rotate about $O$. The forces exerted by the film of gas on the shaft will cause the shaft to whirl about the center $O$. The whirling velocity $\omega$ will be the velocity at which the gas film is carried into the convergent channel, i.e., $\frac{\omega_{s}}{2}$ where $\omega_{s}$ is the angular velocity of rotation of the shaft.

Thus

$$
\omega=\frac{\omega_{s}}{2}
$$

This phenomenon which is known as "half-speed whirl" is often encountered in the bearings of highspeed spindles, particularly under conditions of light loading:

If the shaft is of mass $m$, then there will be a centrifugal force $m \omega^{2} \epsilon$ due to the whirling which acts in the direction of the eccentricity. The pressure in the film of lubricant can only exert forces at right angles to this direction, and if halfspeed whirl is occurring, this pressure is zero; consequently, if the short link is removed, the shaft will move outward along a spiral orbit until it comes into contact with the sleeve. As it moves out, however, compressibility and other effects cause the pressure distribution to become asymmetrical. Under these conditions, the new pressure distribution provides a force which can be resolved into a tangential component and an inwardly directed radial component. In practical bearings, small deviations from perfection, such as variations in the film thickness, may introduce such a radial component even when the shaft is at the center of the bearing. At moderate speeds this radial component may be sufficient to balance the centrifugal force due to whirl.

As the speed is increased, however, the radial component of the force due to the film increases as the first power of the speed, while the centrifugal force due to whirl increases as the square of the speed. Thus it is evident that at some critical speed the centrifugal force should become equal to the radial restoring force, and at this speed the shaft should become unstable. This phenomenon is often observed in practice.

In a perfectly constructed bearing, however, it is evident that a whirl of small amplitude should commence even at a very low shaft speed. This prediction has been verified experimentally by Boeker and Sternlicht [1956].

The tangential force $F_{H}$ which is generated in a bearing of radius $r$, of radial clearance $\delta$ and of length $L$, is given by the equation

$$
F_{H}=\frac{12 \pi \mu r^{3} L \epsilon}{\delta^{3}} \frac{2}{\left(2+n^{2}\right)\left(1-n^{2}\right)^{1 / 2}}\left(\frac{\omega_{s}}{2}-\omega\right) f\left(\frac{L}{r}, n\right)
$$

where

$\mu$ is the viscosity of the gas in lb-sec/in. ${ }^{2}$

$\epsilon$ is the eccentricity in inches

$n$ is the eccentricity ratio

$\omega_{s}$ is the rotational velocity

$\omega$ is the angular velocity of whirl and

$f\left(\frac{L}{r}, n_{b}\right)$ is the end leakage factor which depends on

$$
\frac{L}{r} \text { and } n \text {. }
$$

The first, second, and fourth terms on the righthand side of eq (2) are taken from a paper by Ford, Harris, and Pantall [1957]. The third term, according to Barwell [1956], is introduced in order to account for the effects of whirl. Equation (2) holds only where compressibility effects can be neglected so that the pressure distribution is symmetrical about the line of centers.

The value of the end leakage factor has been determined experimentally by Ford, Harris, and Pantall [1957]. According to the curves in figure 18 of their paper, the value of the end leakage factor in bearings whose length is less than twice the diameter is approximately

$$
f\left(\frac{L}{r}\right) \approx \frac{1}{6.0} \frac{L}{r}
$$

and for small values of $n$, the leakage factor is independent of the eccentricity ratio.

Thus, at small values of the eccentricity ratio, the tangential force in a sleeve bearing whose length is less than twice the diameter is given approximately by the equation

$$
F_{H}=E_{H} \epsilon \approx \frac{2 \pi \mu r^{2} L^{2} \epsilon}{\delta^{3}}\left(\frac{\omega_{s}}{2}-\omega\right)
$$

where $E_{H}$ is the tangential "spring" modulus.

Equation (4) may be written

$$
F_{H}=E_{H} \epsilon \approx \frac{\pi \mu r^{2} L^{2} \omega_{s} \epsilon}{\delta^{3}}\left(1-\frac{2 \omega}{\omega_{s}}\right)
$$

The force $F_{H}$ can be either positive or negative depending on the value of $\omega$. In fact, $F_{H}=0$ when 
$\omega$ is exactly equal to $\frac{\omega_{s}}{2}$. There is no pressure acting on the shaft due to the gas film under this condition. The force $F_{H}$ acts at right angles to the displacement $\epsilon$, and when $\omega<\frac{\omega_{s}}{2}$, then $F_{H}$ acts in the direction of shaft rotation and whirl is accelerated; conversely, when $\omega>\frac{\omega_{s}}{2}$, then $F_{H}$ acts in the opposite direction to shaft rotation and whirl is retarded.

An appreciation of the magnitude of the tangential force in a simple sleeve bearing at an eccentricity $\epsilon$ may be obtained from the following example.

\section{Example 1}

Radius of shaft

Radial clearance

Length of bearing

Viscosity of gas

Angular velocity of shaft

Angular velocity of whirl

0.437 in

$1.50 \times 10^{-3}$ in.

$0.281 \mathrm{in}$.

$2.97 \times 10^{-9} \mathrm{lb}-\mathrm{sec} / \mathrm{in}^{2}$

$1.88 \times 10^{4}$ radians $/ \mathrm{s}$

Zero

$E_{H}=\frac{3.14 \times 2.97 \times 10^{-9} \times(0.437)^{2} \times(0.281)^{2} \times 1.88 \times 10^{4}}{\left(1.5 \times 10^{-3}\right)^{3}}$

$$
E_{H}=784 \mathrm{lb} / \mathrm{in}
$$

Thus at an eccentricity of $0.000393 \mathrm{in} .(0.001 \mathrm{~cm})$ the tangential force is approximately $0.308 \mathrm{lb}$ weight.

It is evident that in order to insure stability at finite speeds of the shaft, the bearing must be designed so that a radial restoring (centering) force $F_{R}$ is generated. If this force is proportional to the eccentricity, it behaves like a spring, and it may be expressed as

$$
Y \epsilon=F_{R}
$$

where $Y$ is the modulus of the "spring" and $\epsilon$ is the eccentricity. An appreciation of the radial spring modulus required in one of the bearings of the miniature turbine may be obtained from the following example.

\section{Example 2}

The effective mass of the shaft in the plane of the most heavily loaded bearing is $0.775 \mathrm{lb}$, and the designed rotational speed is $3,000 \mathrm{rps}$. The corresponding half-speed whirl velocity is $1,500 \mathrm{rps}=9430$ radians/s. Inserting these values in the equation $Y \epsilon=m \omega^{2} \epsilon$ we get

$$
\begin{aligned}
Y & =0.775 \times 9430^{2} / 32.3 \times 12 \\
& =178,200 \mathrm{lb} / \text { in. }
\end{aligned}
$$

At an eccentricity of 0.00039 in., the centrifugal force would be approximately $69.5 \mathrm{lb}$ weight.

\section{External Pressurization}

The necessary restoring force may be generated by means of external pressurization. The sleeve is provided with a number of equally spaced injector holes, as shown in figure 5. Compressed gas is supplied to the injector holes and flows radially outward in the clearance space between the shaft and the sleeve. The shaft is found to float centrally in the sleeve, a well-known effect.

The reason for this effect becomes evident from an examination of a plane circular bearing as shown in the insert of figure 6 . In this figure the relationship between the mean pressure between the plates and the plate separation is shown for various supply pressures. The graphs in figure 6 show the variation of pressure with the separation of the plates for plates of $3 / 8 \mathrm{in}$. diameter. In order to find the corresponding variation of pressure for plates of any diameter, the scaling relationship $h^{2} \sim D$ is used in conjunction with a constant orifice to pad diameter ratio. Accordingly, the graphs shown in figure 7 are drawn for pads $3 / 8$ in. diameter and a plate separation of 0.00165 in., which corresponds to a plate separation of 0.0015 in. with plates $5 / 16$ in. diameter.

As shown in figure 5, the pads in this bearing are not circular but have a rectangular configuration, as shown. It will be assumed that each pad is equivalent to a circular pad $5 / 16$ in. diameter. The mean pressure in the clearance space between a pair of diametrically opposed pads [Sixsmith, Wilson, Birmingham, 1961] is shown in figure 7. Curves a and $b$ show the variation of mean pressure with clearance on the right-hand and left-hand sides of the shaft. Curve $c$ shows the variation of differential pressure with the eccentric displacement of the shaft. Over
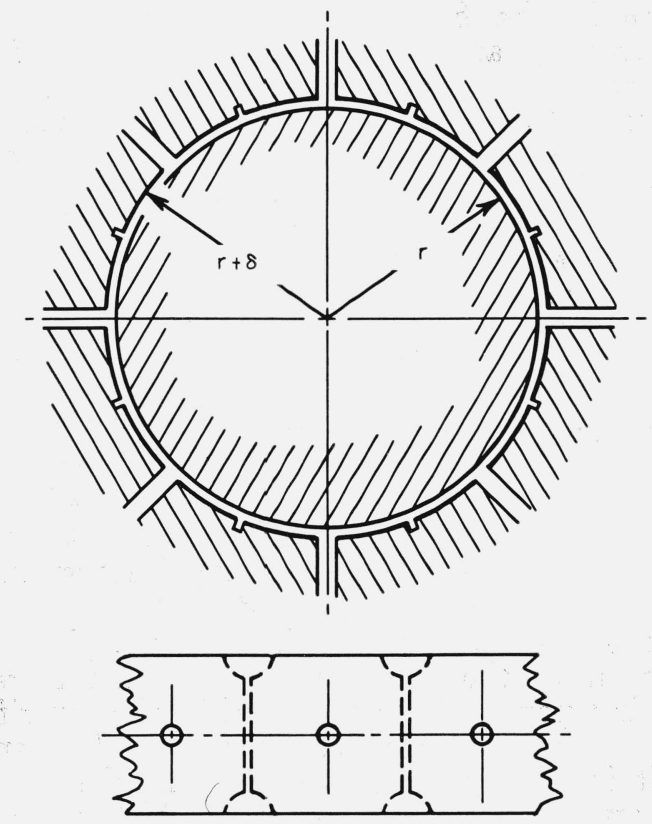

Figure 5. Conventional gas-bearing arrangement. 


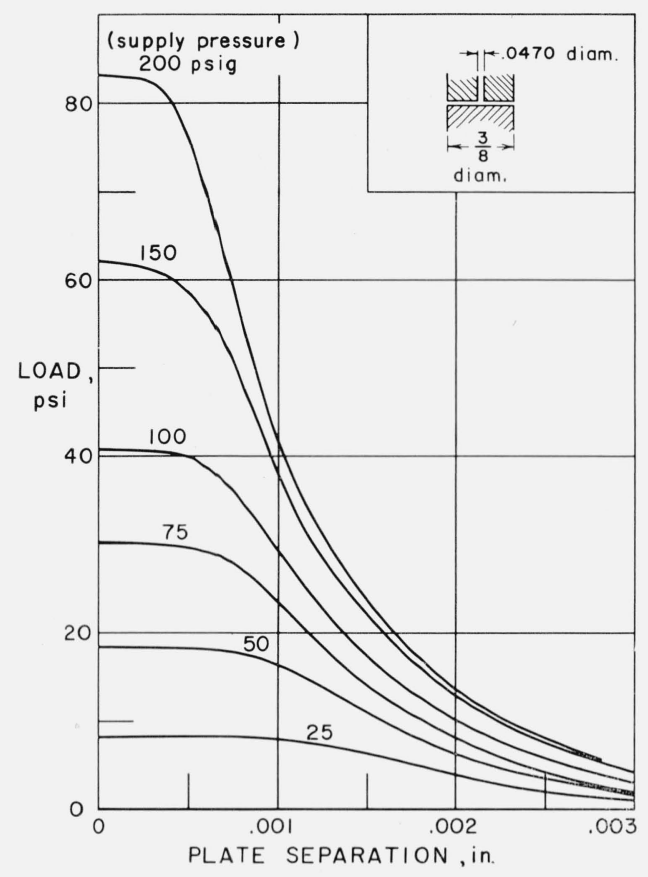

Figure 6. Load versus plate separation curve.

a restricted range the differential pressure (curve c) may be assumed to be linear, thus the corresponding restoring force is also linear, and is directly proportional to the displacement.

If the direction of the radial displacement makes an angle $\gamma$ with a diameter through the center of a pair of diametrically opposed pads, the component of the deflection with respect to the pads is $\epsilon \cos \gamma$. Similarly, the component of the force generated by the pair of diametrically opposed pads in the direction of the displacement is also proportional to $\cos \gamma$. Thus the total restoring force along any radius is given by

$$
Y \epsilon=\frac{1}{2} \frac{\partial P}{\partial \epsilon} A_{1} \epsilon \sum\left[\cos ^{2} \gamma\right]_{2 \pi}^{0}
$$

or

$$
Y \epsilon=\frac{N}{4} A_{1} \frac{\partial P}{\partial \epsilon} \epsilon
$$

where $Y$ is the "spring" modulus of the gas film, $N$ is the number of pads, $A_{1}$ is the area of a pad, and $\frac{1}{2} \frac{\partial P}{\partial \epsilon}$ is the differential pressure due to a single pad as shown in figure 7 .

Example 3.

In the present bearing there are eight pads, and for this particular number of pads,

and (11) becomes

$$
\sum\left[\cos ^{2} \gamma\right]_{2 \pi}^{0}=4
$$

$$
Y_{\epsilon}=2 A_{1} \frac{\partial P}{\partial \epsilon} \epsilon .
$$

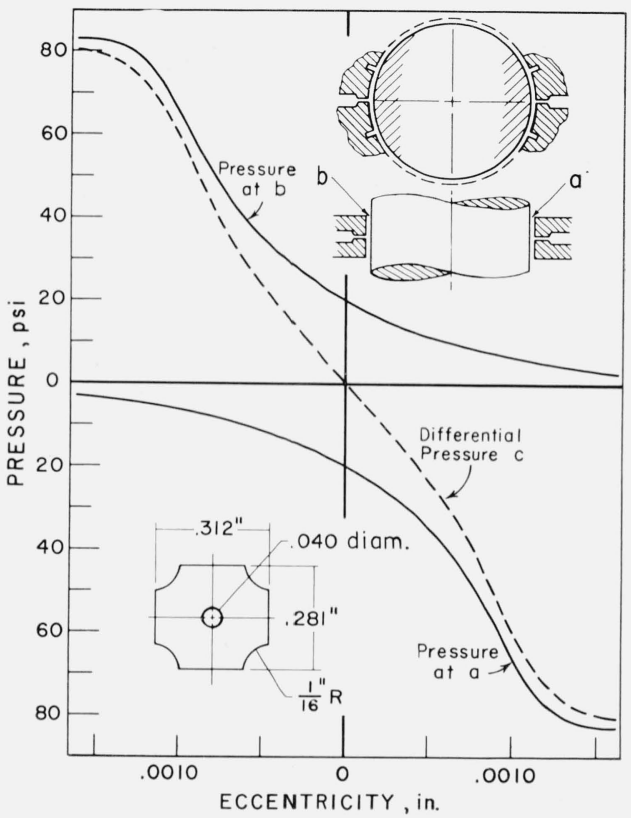

Figure 7. Mean pressure on gas-bearing pads.

The effective area $A_{1}$ of a pad is $0.076 \mathrm{in.}^{2}$, and the slope of curve c at 200 psig supply pressure is 44,000 $\mathrm{lb} / \mathrm{in}^{2} / \mathrm{in}$. Putting these values in (13) we obtain the value of $Y$. Thus

$$
\begin{aligned}
Y & =2 \times 0.076 \times 44,000 \\
& =6,700 \mathrm{lb} / \mathrm{in} .
\end{aligned}
$$

At an eccentric displacement of 0.00039 in., the radial restoring force is $2.61 \mathrm{lb}$. This is less than the corresponding centrifugal force (example 2) by a factor of 27 . Thus it is evident that the radial restoring force, although quite large, is insufficient to prevent the shaft from spiralling outward until it touches the bearing. It is then necessary to devise a method whereby the centrifugal force can be reduced to a value below the available radial restoring force in order to obtain a stable bearing. This can be accomplished by reducing the shaft whirl speed. The method used to reduce whirl speed is presented in sec. 6 .

\section{Analysis of Turbine Rotor Instabilty}

In an early design of a turbine [Sixsmith, 1959a] in which the turbine was mounted on a flexible shaft, it was discovered that a hydrodynamic excitingforce can be generated by the turbine rotor. At a certain critical pressure of the gas supply, a whirling of the shaft would commence. The shaft could be made to whirl even when it was prevented from rotating. In order to suppress the whirl it was necessary to provide damping in the form of oil-filled dash-pots. 


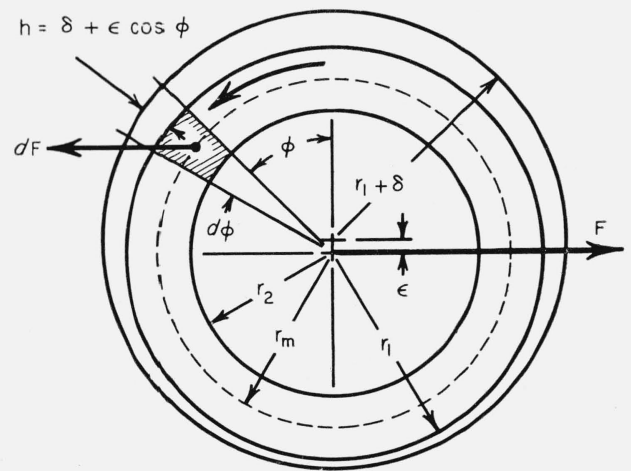

Figure 8. Force distribution on turbine rotor.

An explanation of this phenomenon is as follows. The effect appears to be due to forces which are generated by the variation of blade-tip leakage with rotor eccentricity. Consider the turbine rotor shown in figure 8. If the rotor is displaced downwards as shown, the tip leakage is reduced around the lower half of the rotor and correspondingly increased around the upper half. Around the lower half of the rotor the tip leakage is less, hence an increased thrust is developed on the blades. This force is to the right for counterclockwise rotation. Around the top half, tip leakage increases and the thrust on the blades is reduced. This can also be regarded as an added component of force to the right. Consequently there is a net force to the right acting on the rotor as a whole. This force is at right angles to the displacement, and therefore, it is in the same direction as the hydrodynamic force which is generated in a simple sleeve bearing.

An estimate of its magnitude may be made if we assume that the loss of torque due to tip leakage is directly proportional to the tip clearance, i.e.,

$$
d T=\frac{T d \phi}{2 \pi}\left[1-\frac{2 \pi r_{1} h v_{1}}{\pi v_{2}\left(r_{1}^{2}-r_{2}^{2}\right)}\right]
$$

where

$d T=$ element of torque acting on the blades between $\phi$ and $\phi+d \phi$

$T=$ total torque acting on the rotor-in. $\mathrm{lb}$

$r_{1}=$ tip radius - inches

$r_{2}=$ root radius - inches

$h=$ mean tip clearance between $\phi$ and $\pi+d \phi$ inches

$v_{1}=$ axial component of velocity of gas through the tip clearance in./s

$v_{2}==$ axial component of velocity of gas through the annulus - in./s between $r_{1}$ and $r_{2}$.

If the rotor is deflected downwards a distance $\epsilon$ the tip clearance $h$ at a point which makes an angle $\phi$ with the direction of the displacement is given by

$$
h=\delta+\epsilon \cos \phi .
$$

The horizontal force $d F$ to the right which acts on the small segment of blading lying between $\phi$ and $\phi+d \phi$ is given by

$$
d F=\frac{d T}{r_{m}} \cos \phi
$$

where $r_{m}=$ mean radius of rotor blades. Thus

$$
d F=\frac{T d \phi}{2 \pi r_{m}}\left[1-\frac{2 \pi r_{1} h v_{1}}{\pi\left(r_{1}^{2}-r_{2}^{2}\right) v_{2}}\right] \cos \phi .
$$

Substituting $(\delta+\epsilon \cos \phi)$ for $h$ we have

$$
d F=\frac{T d \phi}{2 \pi r_{m}}\left[1-\frac{2 r_{1} v_{1}}{\left(r_{1}^{2}-r_{2}^{2}\right) v_{2}}(\delta+\epsilon \cos \phi)\right] \cos \phi .
$$

On integrating between $O$ and $2 \pi$, all terms will vanish except the one containing $\cos ^{2} \phi$. thus we have

$$
\begin{aligned}
F & =\frac{T \epsilon}{2 \pi r_{m}} \times \frac{2 r_{1} v_{1}}{\left(r_{1}^{2}-r_{2}^{2}\right) v_{2}}\left(\frac{1}{2} \sin \phi \cos \phi+\frac{1}{2} \phi\right)_{0}^{2 \pi} \\
& =\frac{T \epsilon r_{1} v_{1}}{\pi r_{m}\left(r_{1}^{2}-r_{2}^{2}\right) v_{2}}\left(\frac{1}{2} \phi+\frac{1}{4} \sin 2 \phi\right)_{0}^{2 \pi} \\
& =\frac{T \epsilon r_{1} v_{1}}{r_{m}\left(r_{1}^{2}-r_{2}^{2}\right) v_{2}}
\end{aligned}
$$

which may be written as

$$
F=k T \epsilon
$$

where

$$
k=\frac{r_{1} v_{1}}{r_{m}\left(r_{1}^{2}-r_{2}^{2}\right) v_{2}} .
$$

Thus it appears that the whirl-inducing force is proportional to the product of the torque and the eccentricity, but is independent of tip clearance as long as the assumption that the blade passage efficiency is inversely proportional to tip clearance is valid. Since for a given eccentricity it is proportional to the torque, it is capable of inducing a whirl even when the shaft is prevented from rotating.

In the miniature turbine the value of the torque at $3000 \mathrm{rps}$ and $3 \mathrm{kw}$ of power is $1.406 \mathrm{lb}$-in. The values of $r_{1}, r_{m}$, and $r_{2}$ are $0.436,0.397$, and 0.36 in. Since the blade outlet angle is $30^{\circ}$, the value of $\frac{v_{1}}{v_{2}}$ is 2 , approximately. Putting these values in (17) we have

$$
F=\frac{1.406 \times 0.436 \times 2 \times \epsilon}{0.397\left(0.436^{2}-0.360^{2}\right)}
$$

or

$$
F=51.15 \epsilon \mathrm{lb} \text {. }
$$

Thus at an eccentricity of $0.00039 \mathrm{in}$. the tangential force is $0.02 \mathrm{lb}$ weight. 


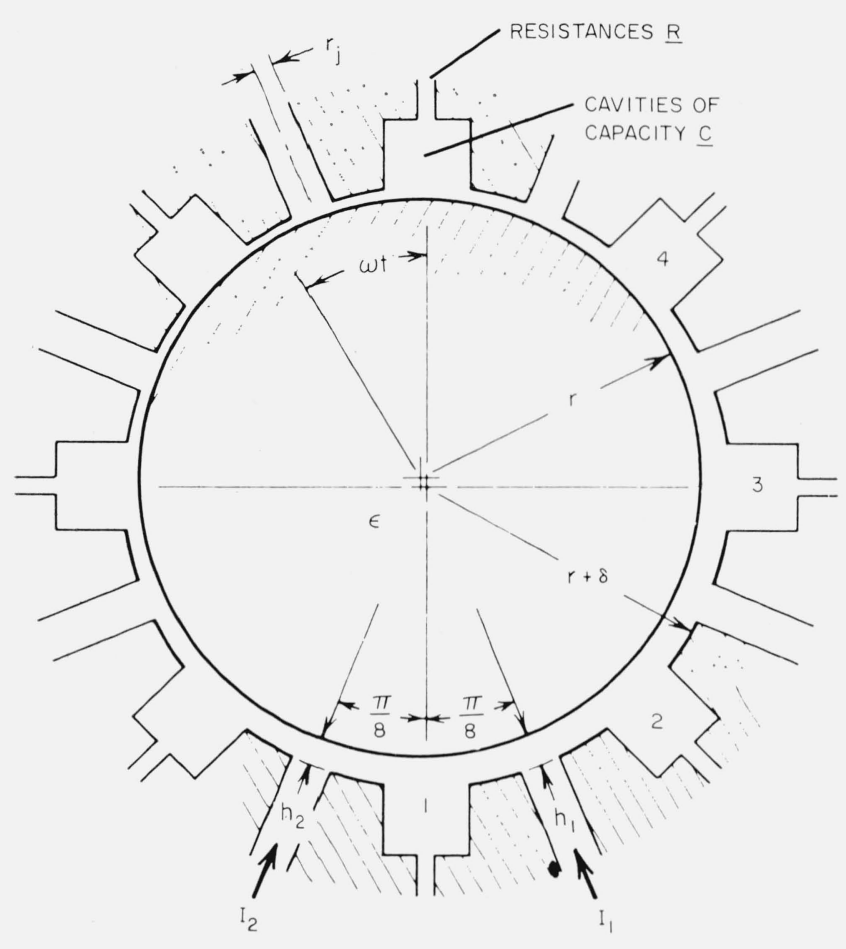

Figure 9. Gas bearing with stabilizing cavities.

\section{The Analysis of a Method of Stabilizing a Gas-Lubricated Bearing}

In order to ensure stability, it is necessary to reduce the angular velocity of whirl until the centrifugal force becomes less than the radial restoring force. A reduction in the angular velocity of whirl may be effected if the bearing is designed to generate a force which acts in the opposite direction to the hydrodynamic exciting forces due to the bearing and the turbine rotor.

A bearing designed to produce this stabilizing force is shown in figure 9 . In addition to the injector holes, as shown in figure 5, the shaft is surrounded by a number of pneumatic phase shift networks which are spaced midway between the injector holes. Each network consists of a cavity which acts as a pneumatic capacitor and a leak orifice which acts as a pneumatic resistor. A stream of gas flows into each cavity from the adjacent injector holes, and escapes from the cavity via the leak orifice. The pressure in the cavity is determined by the flow rate and the resistance of the orifice. In the event of a whirling of the shaft, the flow of gas into each cavity is modulated, the modulation being in phase with the eccentricity vector. As a consequence of the modulated inflow, the pressure in the cavity is likewise modulated, but by virtue of the finite volume of the cavity, the phase of the pressure is lagging with respect to the phase of the current and the eccentricity. The force on the area of shaft facing the cavity, being in phase with the pressure, is likewise lagging with respect to the current and eccentricity vectors. This force can be resolved into components whose directions are radial and tangential with respect to the eccentricity vector. The tangential component acts in the opposite direction to the hydrodynamic force due to the bearing and the turbine rotor. By suitable design of the bearing, the tangential component of force can be made to exceed the exciting forces so that the net tangential force is opposed to the tangential motion of whirl, and consequently, the whirling motion should be damped out.

The radial component acts in the opposite direction to the centering force due to the injector holes, and therefore it reduces the load carrying capacity of the bearing. This is inherent in the design and cannot be avoided.

As shown in figure 9, a shaft of radius $r$ is surrounded by a sleeve of internal radius $r+\delta$. Gas from eight injector holes each of radius $r_{j}$, equally displaced around the circumference of the sleeve, flows into eight cavities each of capacity $C$. The majority of the gas escapes from each cavity through a leak orifice of resistance $R$. The remainder escapes at the ends of the bearing, but it will be assumed that this portion is negligible. Each cavity and leak
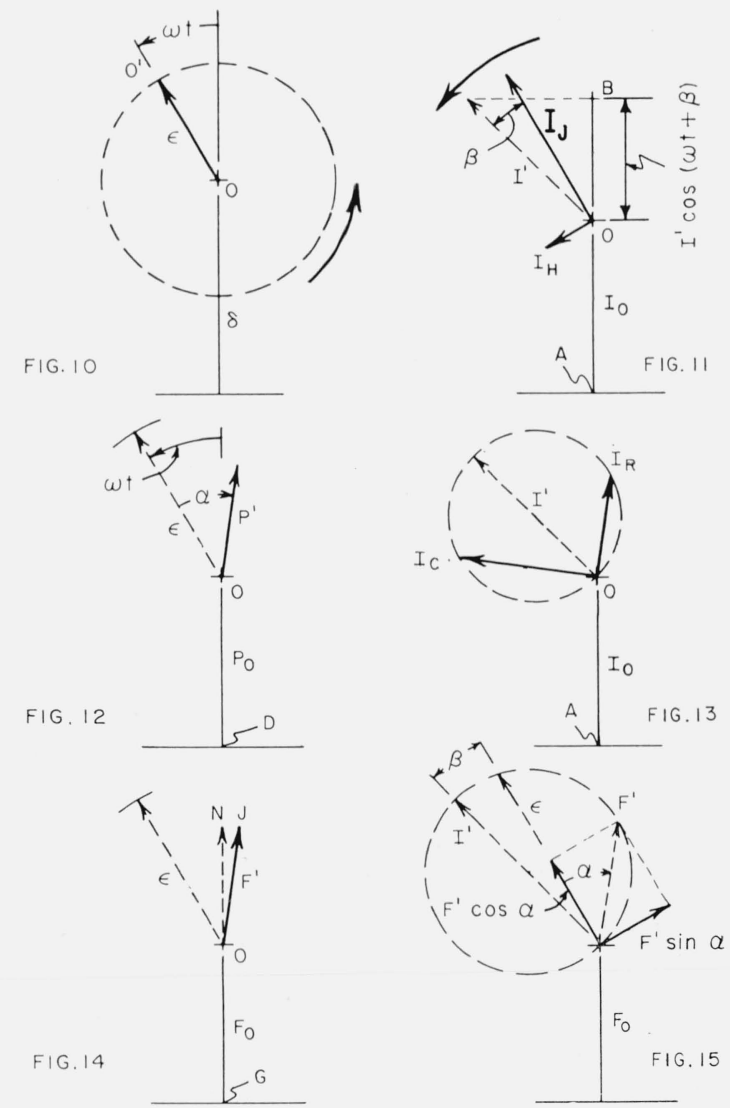

Figures 10-15 (inc.) Vector diagrams illustrating the action of a stabilizing cavity. 
behaves like an electrical resistance-capacity network: the capacity of a cavity being defined by the equation

$$
C \frac{\partial P}{\partial t}=V \frac{\partial \rho}{\partial t}=V \frac{\rho}{P} \frac{\partial P}{\partial t}=I_{c} \mathrm{lb} / \mathrm{s}
$$

and the resistance of a leak by

$$
\frac{P}{R}=I_{L} \mathrm{lb} / \mathrm{s}
$$

where $V$ is the volume of the cavity, $\rho$ is the density of the gas in the cavity, $P$ is the pressure in the cavity, $I_{c}$ is the current of gas accumulating in the cavity, and $I_{L}$ is the current flowing through the leak: $t$ is time.

A cavity receives its gas supply from both of the adjacent injector holes. Suppose now that the shaft is whirling with an eccentricity $\epsilon$ and a counterclockwise angular velocity $\omega$. The motion of the center of the shaft may be represented by a complex vector $\epsilon$ rotating counterclockwise with the same angular velocity (fig. 10).

Referring again to figure 9 , it will be seen that the current of gas $I$ entering cavity 1 will be given by

$$
\begin{aligned}
I & =1 / 2 I_{1}+1 / 2 I_{2} \\
& =\pi r_{j} v \rho_{1}\left(h_{1}+h_{2}\right)
\end{aligned}
$$

where $v$ is the velocity of the gas at the edges of the injector holes and $\rho_{1}$ is its density. The values of the clearance at the injector holes adjacent to cavity 1 are given by

$$
h_{1}=\delta+\epsilon \cos \left(\omega t-\frac{\pi}{N}\right)
$$

and

$$
h_{2}=\delta+\epsilon \cos \left(\omega t+\frac{\pi}{N}\right)
$$

where $N$ is the number of cavities.

The time $t$ is assumed to be zero when $\epsilon$ is pointing vertically downward through the center of cavity 1 .

Equation (21) may now be written

$$
\begin{aligned}
I & =\pi r_{j} v \rho_{1}\left\{\delta+\epsilon \cos \left(\omega t-\frac{\pi}{N}\right)+\delta+\epsilon \cos \left(\omega t+\frac{\pi}{N}\right)\right\} \\
& =\pi r_{j} v \rho_{1}\left\{2 \delta+2 \epsilon \cos \omega t \cos \frac{\pi}{N}\right\} .
\end{aligned}
$$

The current flowing into cavity 1 thus consists of a steady component $\hat{I}_{0}$ which is given by

$$
I_{0}=2 \pi r_{j} v \rho_{1} \delta
$$

and an alternating component $I_{j}$ which rotates in phase with the eccentricity vector $\epsilon$ and whose instantaneous value is given by

$$
I_{j} \cos \omega t=2 \pi r_{j} v \rho_{l} \epsilon \cos \omega t \cos \frac{\pi}{N} .
$$

In the above analysis, the effects of shaft rotation and whirl have been neglected. These effects influence the flow rate into the cavity. Referring to figure 9 , it is seen that the clearance between the shaft and the bearing on the right-hand side of the cavity is greater than the clearance on the left-hand side. As a consequence of the rotation of the shaft, more gas is carried out of the cavity on the righthand side than is carried in on the left-hand side. Thus there is a net flow of gas out of the cavity. An estimate of the value of this component of current may be made if it is assumed that the principle of superposition may be applied; i.e., that the velocity of the gas at any point in the film is the vector sum of the radial velocity from the injector holes and the tangential velocity due to hydrodynamic action.

The instantaneous value of the hydrodynamic component of current $I_{a}$ is given by

$$
\begin{aligned}
I_{a} \sin \omega t= & \frac{\omega_{s}}{2} L_{c} r \rho_{2}\left(h_{2}-h_{1}\right) \\
& =\frac{\omega_{s}}{2} L_{c} r \rho_{2}\left\{\left[\delta+\epsilon \cos \left(\omega t+\frac{\pi}{N}\right)\right]\right. \\
& \left.-\left[\delta+\epsilon \cos \left(\omega t-\frac{\pi}{N}\right)\right]\right\} \\
& =-\omega_{s} L_{c} r \rho_{2} \epsilon \sin \omega t \sin \frac{\pi}{N}
\end{aligned}
$$

where $l_{a}$ is the alternating flow of current, $\omega_{s}$ is the angular speed of the shaft, $r$ is the radius of the shaft, $L_{c}$ is the effective length of the cavity, and $\rho_{2}$ is the density of the gas in the clearance space.

Referring again to figure 9 , it is seen that as a consequence of the whirling motion, the shaft is approaching the cavity and therefore the volume of the cavity is decreasing. This may be regarded as a current $\tilde{I}_{W}$ flowing into the cavity, the volume of the cavity being regarded as constant. The instantaneous value of the current is given by the equation

$$
I_{W} \sin \omega t=2 \omega L_{c} r \rho_{2} \epsilon \sin \omega t \sin \frac{\pi}{N}
$$

where $I_{W}$ is the alternating flow of current and $\omega$ is the angular velocity of whirl.

Thus the resultant current $I_{H}$ due to the effects of shaft rotation and whirl is given by the equation

$$
I_{H} \sin \omega t=-L_{c} r \rho_{2} \epsilon \omega_{s}\left(1-\frac{2 \omega}{\omega_{s}}\right) \sin \omega t \sin \frac{\pi}{N}
$$

This current may be represented by the vector $\mathbf{I}_{\mathbf{H}}$ (fig. 11) which rotates $90^{\circ}$ ahead of the eccentricity vector $\epsilon$. 
The resultant current flowing into the cavity is represented by the vector $\mathbf{I}^{\prime}$. It is seen that

$$
\mathbf{I}^{\prime}=I_{j} \sec \beta .
$$

The direct current $I_{0}$ flowing into the cavity may be represented by vertical line $O A$, figure 11 , and the instantaneous value of the alternating current by the vertical line $O B$. This is the projection on a diameter through cavity 1 of the alternating current $I^{\prime}$ which rotates ahead of the eccentricity vector $\epsilon$ with a phase angle $\beta$.

The value of $\beta$ is given by

$$
\tan \beta=\frac{I_{H}}{I_{J}}=\frac{L_{c} r \rho_{2} \omega_{s}}{2 \pi r_{j} v \rho_{1}}\left(1-\frac{2 \omega}{\omega_{s}}\right) \tan \frac{\pi}{N} .
$$

The steady current $I_{0}$ flowing through the resistance $R$ generates a pressure given by

$$
P_{0}=\mathbf{I}_{0} R .
$$

This is represented by the vertical line $O D$, figure 12 .

The alternating current generates an alternating pressure component

$$
P^{\prime}=I^{\prime} Z=I_{j} Z \sec \beta=\frac{I_{j} R \sec \beta}{\sqrt{1+\omega^{2} C^{2} R^{2}}}=\frac{I_{j} R \sec \beta}{\sqrt{1+\tan ^{2}(\alpha+\beta)}}
$$

where $Z$ is the impedance of the resistance-capacity network. With respect to the alternating component of current, the capacity and resistance are effectively in parallel and, consequently, the phase of the pressure lags behind that of the current and the eccentricity. The phase angle $\alpha$ is given by

$$
\tan (\alpha+\beta)=\omega R C .
$$

The currents due to the alternating pressure $P^{\prime}$ are represented vectorially in figure 13 . The vector $\mathbf{I}_{\mathbf{R}}$ represents the alternating current flowing in the resistance $R$, and the vector $\mathbf{I}_{\mathbf{C}}$ represents the alternating current flowing in the capacity. The values of $\mathbf{I}_{\mathbf{R}}$ and $\mathbf{I}_{\mathbf{C}}$ are given by the equations

$$
\mathbf{I}_{\mathbf{R}}=\frac{P^{\prime}}{R}
$$

and

$$
\mathbf{I}_{\mathbf{c}}=P^{\prime} \omega C=P^{\prime} \omega V \frac{\rho}{P} .
$$

The resultant of these two currents must be equal to and in phase with the alternating current $I^{\prime}$ flowing into the cavity.

The pressures $P_{0}$ and $P^{\prime}$ acting on the effectiv $\mathrm{e}^{1}$ area $A_{2}$ of the shaft, which forms one wall of the cavity, generate forces

$$
F_{0}^{\prime}=A_{2} P_{0}
$$

and

$$
F^{\prime}=A_{2} P^{\prime}
$$

These forces may be represented by the vertical vector OG (fig. 14) and the alternating vector $\mathbf{O J}$, respectively. The alternating vector $\mathbf{F}^{\prime}$ may be resolved into a component $\mathbf{F}^{\prime} \cos \alpha$ which is in phase with the eccentricity and a component $\mathbf{F}^{\prime}$ sin $\alpha$ which is lagging $90^{\circ}$ behind the eccentricity (fig. 15).

These forces are generated by a single cavity and act along the radius from the center of the shaft through the center of that cavity. With a symmetrical array of cavities, the resultant of the steady forces $F_{0}$ will be zero; this, however, is not true of the alternating components.

The resultant force on the shaft at any instant is found by adding vectorially the instantaneous values of the forces due to each of the cavities.

The instantaneous value of the force due to cavity 1 is

$$
\mathbf{F}^{\prime} \cos (\omega t-\alpha) \text {. }
$$

This is the projection of the alternating vector $\mathbf{F}^{\prime}$ along a diameter through the center of the cavity. At a given instant it is represented in magnitude and direction by the vertical line $O N$ as shown in figure 14 .

The polar diagram of the force due to each of the eight cavities is shown in figure 16 . At an instant of time $t$, the vectors numbered 1 to 8 represent the forces due to the corresponding cavities.

The polar diagram represents a field of force rotating with the angular velocity of whirl. This is illustrated in figure 17, which shows the values of the eight forces due to the cavities when $t$ has increased to $t+\Delta t$. These forces can be combined into a resultant which by symmetry will lie along OJ. The magnitude of this force may be written as $F_{S}$

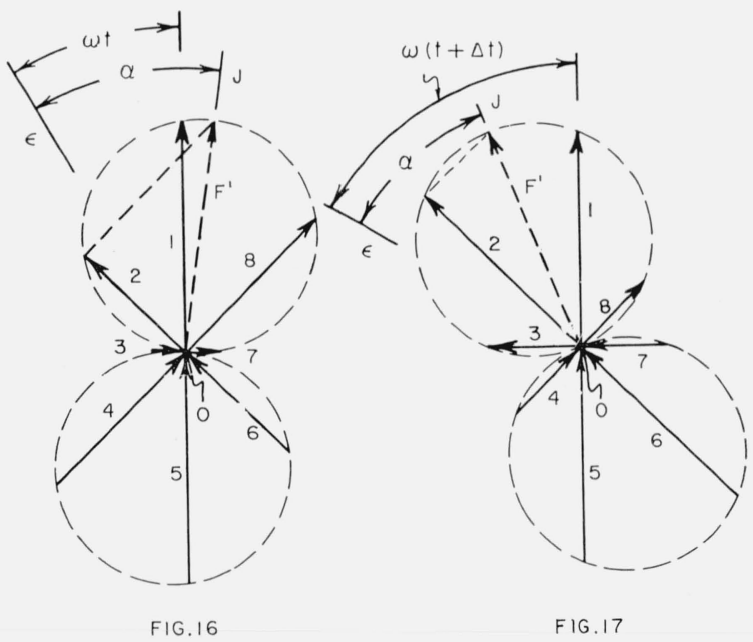

Figures 16 and 17. Polar diagrams of force generated by stabilizing cavities.

\footnotetext{
1 Experimental testing has shown $\mathrm{A} / 2$ to be a conservative estimate for the value of $A_{2}$ as illustrated in figure 20 .
} 
and is given by

$$
F_{S}=\frac{N}{2} \mathbf{F}^{\prime}
$$

Substituting from (36), (31), (29), and (24) this becomes

$$
F_{S}=N \pi A_{2} r_{j} v \rho_{1} Z \epsilon \cos \frac{\pi}{N} \sec \beta
$$

or

$$
F_{S}=G Z_{\epsilon}
$$

where

$$
G=N \pi A_{2} r_{j} v \rho_{1} \cos \frac{\pi}{N} \sec \beta .
$$

$G$ is a constant which depends on the design of the bearing and the speed of the shaft. The alternating vector GZ $\epsilon$ may be resolved into components $G Z \epsilon$ $\cos \alpha$ in phase with the eccentricity vector or GZE $\sin \alpha$ lagging $90^{\circ}$ behind the eccentricity vector. The component of force $G Z_{\epsilon} \cos \alpha$ due to the stabilizing cavities is in the same direction as the centrifugal force due to whirl and in the opposite direction to the centering force due to the injector holes defined in (11). Consequently, the centering force is reduced to $Y \epsilon-G Z \epsilon \cos \alpha$. Whirl can set in at an angular velocity given by

$$
m \omega^{2} \epsilon=Y \epsilon-G Z \epsilon \cos \alpha ;
$$

i.e.,

$$
\omega=\left(\frac{Y-G Z \cos \alpha}{m}\right)^{1 / 2}
$$

These forces are represented as vectors in figure 18 .

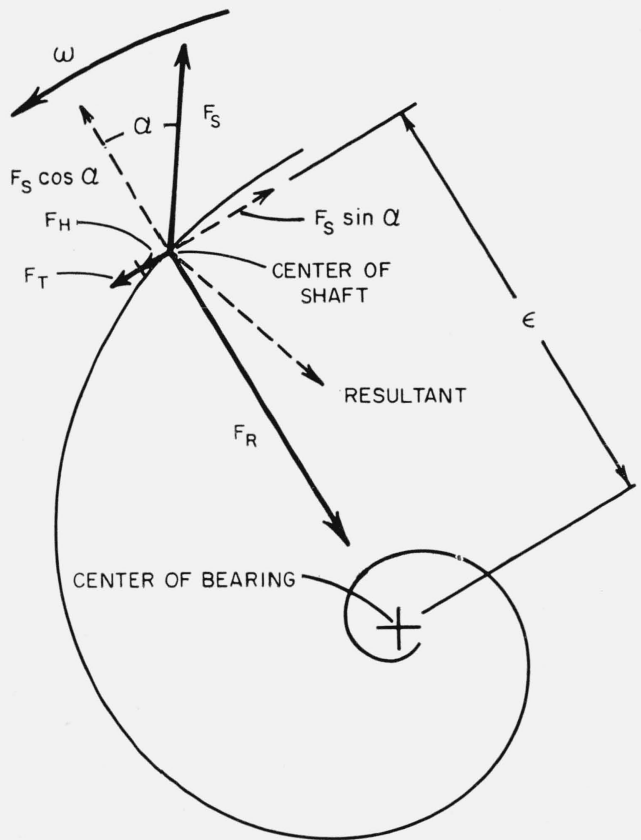

FIGURE 18. Illustration of forces on shaft.
The component $G Z_{\epsilon} \sin \alpha$ provides a force at right angles to the eccentricity vector and acts in the opposite direction to the forces due to hydrodynamic action or excitation in the bearing and the turbine rotor $F(17)$. This is the damping force which is required to provide stability. Provided it is greater than the sum of the bearing and turbine rotor excitations, the direction of the resultant of all the forces lies behind the line of centers, and any whirl, such as might be excited by an external shock, is attenuated until its amplitude becomes zero.

An estimate of the magnitude of the hydrodynamic force due to the bearing may be made as follows. The bearing is regarded as a simple sleeve bearing in which the shaft rotates with an angular velocity $\omega_{s}$ and a whirl velocity $\omega$. The value of the whirl velocity $\omega$ is given by (43). The hydrodynamic force which is generated at an eccentricity $\epsilon$ can be calculated with the aid of (5). The force thus calculated may be expressed in the form

$$
E_{H} \epsilon=F_{H}
$$

for small values of the eccentricity ratio $\epsilon / \delta$. $E_{H}$ is a constant whose value depends on the design of the bearing. In the above calculation no account is taken of the disturbance in the flow pattern due to the various holes and slots in the surface of the bearing. These have the effect of reducing the pressures due to hydrodynamic action and therefore the calculated value of the hydrodynamic force $E_{H} \epsilon$ is an overestimate. Thus it errs on the safe side. The force $E_{H} \epsilon$ is represented vectorially in figure 18 .

The rotor excitation $k T$ is given by (17). This applies however to the force in plane of the rotor; hence, the value must be multiplied by some factor $k_{1}$, depending on the overhang, in order to give the force in the plane of the bearing. This force may thus be expressed in the form

where

$$
F_{T}=k^{\prime} T \epsilon
$$

$$
k^{\prime}=k_{1} k \text {. }
$$

This force is shown vectorially in figure 18 . The term $k^{\prime} T$ can be regarded as the rotor excitation reduced to the plane of the bearing. Referring to figure 19 , it is seen that the excitation due to the rotor is greater in the inner bearing than it is in the outer bearing. For the inner bearing the value of $k_{1}$ is given by

$$
k_{1}=\left(\frac{L_{5}}{L_{2}+L_{4}}\right) .
$$

The stabilizing force $G Z_{\epsilon} \sin \alpha$ which is generated by the bearing must be greater than the sum of the two excitations outlined above if the shaft is to be stable.

The condition required for dynamic stability is thus given by

$$
G Z \epsilon \sin \alpha>k^{\prime} T \epsilon+E_{H} \epsilon .
$$




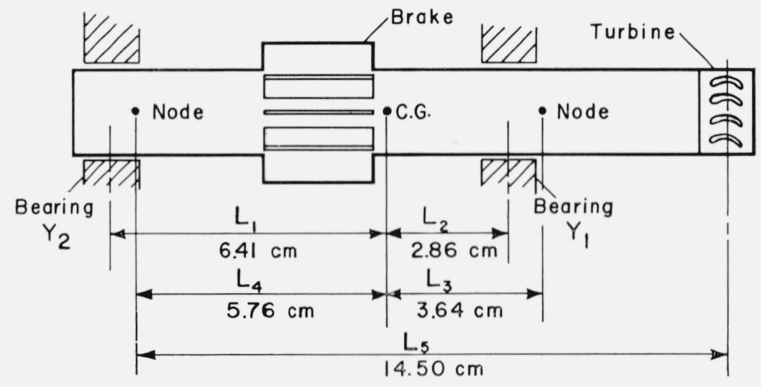

Figure 19. Shaft resonant system.

Provided this condition is satisfied, the magnitude of $\epsilon$ will progressively decrease to zero and the vector diagram in figure 18 will shrink into the origin. On the other hand, if the sum of the excitation is greater than the damping term, the vector diagram will progressively expand until $\epsilon$ becomes equal to the radial clearance at which point the bearing may be destroyed.

In the above analysis the conditions which determine the dynamic stability of the bearing have been outlined. However, the bearing must also possess static stability, i.e., it must be capable of supporting a radial load, such as the weight of the shaft if it were horizontally supported. In this case, $\omega$ becomes zero and if $\omega_{s}$ is also zero then $\beta$ is zero, and since tan $(\alpha+\beta)=\omega C R$, the phase angle $\alpha$ also takes the value of zero. The stabilizing force $G Z \epsilon \sin \alpha$ thus reduces to zero, and the decentering force $G Z \epsilon \cos \alpha$ reduces to the value GRt. The centering force now becomes

$$
F_{A}=(Y-G R) \epsilon,
$$

and it is evident that $G R$ should be less than $Y$ in order to ensure static stability. If $G R$ should be greater than $Y$, the shaft will be pushed to one side of the bearing. It is evident that the value of the leak resistance must be carefully chosen; it must be large enough to ensure dynamic stability; if it is made too large, however, the shaft will not float.

If we write

$$
\frac{G R}{Y}=B
$$

then the static stability condition will be satisfied if $B$ is less than one. The static centering force may now be written

$$
F_{A}=Y \epsilon(1-B) \text {. }
$$

The value of the leak resistance is given by

$$
R=\frac{P^{\prime}}{I_{R}}=\frac{B Y_{\epsilon}}{G \epsilon} .
$$

Substituting for $G$ from (41) we get

$$
R=\frac{B Y}{N \pi A_{2} r_{j} v \rho_{1} \cos \frac{\pi}{N} \sec \beta} .
$$

In the present bearing, $B$ was chosen to be 0.75 , and consequently, the static load carrying capacity is $0.25 Y \epsilon$. The area of the shaft $A_{2}$ which faces each cavity is estimated to be as shown in figure 20 . The value of $\beta$ is given by (29). As a first approximation it may be assumed that $\beta=0$ and sec $\beta=1$. The flow velocity $v$ is assumed to be sonic at the throat formed by the annulus $2 \pi r_{j} \delta$; i.e., the discharge coefficient is assumed to be unity. The diameter of the leak orifices may be calculated with the aid of this equation. The discharge coefficient for a circular orifice is approximately 0.6.

Since the shaft is supported on two bearings, each of which behaves like a spring, it has two principal modes and frequencies of whirl. The calculation of the modes and frequencies of such a resonant system is described in standard textbooks on mechanical vibrations. However, for the convenience of the reader, the procedure in this specinc application is outlined below.

The shaft of the miniature turbine and its supports are shown schematically in figure 19 , where the following symbols and the corresponding values are used.

$m=$ Mass of shaft $-\mathrm{lb}$ mass

$r_{g}=$ Radius of gyration of shaft about the center of gravity - in.

$Y_{1}=$ Stiffness of gas film in outer bearing- $\mathrm{lb} / \mathrm{in}$.

$Y_{2}=$ Stiffness of gas film in inner bearing $-\mathrm{lb} / \mathrm{in}$.

$L_{1}=$ Distance of outer bearing from center of gravity - in.

$L_{2}=$ Distance of inner bearing from center of gravity-in.

It is assumed that the two radial spring constants are equal, i.e.,

$$
Y_{1}=Y_{2}=Y-G Z \cos \alpha .
$$

In this equation, a value for $\alpha$ which should provide the optimum damping is required. This value is given by the equation

$$
\alpha=\frac{90^{\circ}-\beta}{2}
$$

where

$$
\tan \beta=\frac{I_{H}}{I_{j}}
$$

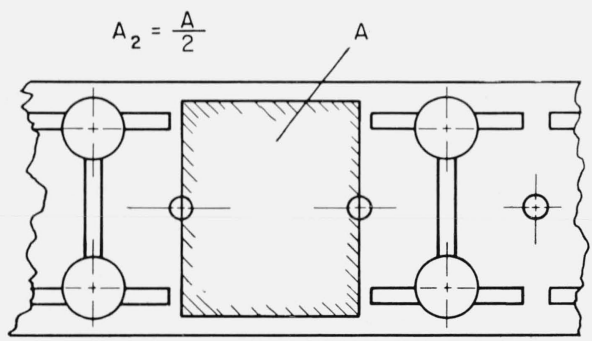

Figure 20. Effective area facing cavity. 
as shown in figure 11. But $I_{H}$ cannot be evaluated until the value of $\omega$ is known, and this depends on the value of $\cos \alpha$.

This difficulty may be circumvented if it is assumed as a first approximation that the angle $\beta$ is zero and the angle $\alpha$ is $45^{\circ}$. With these values of $\alpha$ and $\beta, Z$ becomes equal to $R / \sqrt{2}$ and (54) becomes

$$
Y_{1}=Y_{2}=Y-\frac{1}{2} G R \text {. }
$$

In the present calculation the value of $R$ is arbitrarily chosen so that

$$
G R=\frac{3}{4} Y
$$

The two frequencies of oscillation are given by the equations

$$
\omega_{1}^{2}=\frac{1}{2}(a+c)+\sqrt{\frac{1}{4}(a-c)^{2}+\left(\frac{b}{r}\right)^{2}}
$$

and

$$
\omega_{2}^{2}=\frac{1}{2}(a+c)-\sqrt{\frac{1}{4}(a-c)^{2}+\left(\frac{b}{r}\right)^{2}}
$$

where

$$
\begin{aligned}
& a=\frac{Y_{1}+Y_{2}}{m}, \\
& b=\frac{L_{2} Y_{2}-L_{1} Y_{1}}{m}, \\
& c=\frac{L_{1}^{2} Y_{1}+L_{2}^{2} Y_{2}}{m r_{g}^{2}} .
\end{aligned}
$$

Equations (59) and (60) are approximate and do not include the gyroscopic effects associated with rotation of the shaft. However, such effects can be ignored for the case considered here.

The distances of the nodes from the center of gravity are given by the equations

$$
\begin{aligned}
& L_{3}=\frac{b}{\omega_{1}^{2}-a}, \\
& L_{4}=\frac{b}{\omega_{2}^{2}-a} .
\end{aligned}
$$

The positions of the two nodes are illustrated in figure 19. From the positions of the nodes it is seen that the predominant amplitudes of whirl in the inner and outer bearings occur at the lower and higher frequencies of whirl.

These values of $\omega_{1}$ and $\omega_{2}$ may now be employed in (31) and (54) in order to find more precise values of $Y_{1}$ and $Y_{2}$. From the revised values of $Y_{1}$ and $Y_{2}$, a more refined calculation of the values of $\omega_{1}$ and $\omega_{2}$ may be made. However, in view of the many uncertainties in the computation, the first approximation is usually sufficiently accurate for practical purposes.

The value of the phase angle $\beta$ may now be calculated with the aid of (29). The optimum value of the phase angle $\alpha$ is given by (55).

The values of the stabilizing cavities may now be calculated. We have

$$
\tan (\alpha+\beta)=\omega C R=\frac{\omega V \rho R}{P}
$$

or

and

$$
V_{1}=\frac{\tan (\alpha+\beta) P}{\omega_{1} \rho R}
$$

$$
V_{2}=\frac{\tan (\alpha+\beta) P}{\omega_{2} \rho R}
$$

where $\omega_{1}$ and $\omega_{2}$ are the whirl angular velocities of the outer and inner bearings, $V_{1}$ and $V_{2}$ are the corresponding volumes of the stabilizing cavities, and $\rho$ is the density of the gas at $P$ units of pressure.

Referring to figures 12 and 11 , it is seen that the vectors of pressure and current are given by the equations

and

$$
\mathbf{P}^{\prime}=\mathbf{I}^{\prime} \mathbf{Z}
$$

$$
I^{\prime}=I_{j} \sec \beta \text {. }
$$

The value of $Z$ is given by the equation

$$
Z=\frac{R}{\left\{1+\tan ^{2}(\alpha+\beta)\right\}^{1 / 2}} .
$$

The value of the force $F^{\prime}$, figure 14 , due to a single cavity is given by the equation

$$
F^{\prime}=A_{2} P^{\prime}
$$

where $A_{2}$ is the effective area of the shaft (fig. 20) which forms part of the walls of the cavity. The value of the force $G Z_{\epsilon}$ (fig. 18) is given by the equation

$$
G Z_{\epsilon}=\frac{N}{2} F^{\prime} .
$$

Thus the value of $G Z \epsilon$ is given by

$$
G Z_{\epsilon}=\frac{N A_{2} R I_{j} \sec \beta}{2\left\{1+\tan ^{2}(\alpha+\beta)\right\}^{1 / 2}} .
$$

The corresponding tangential and radial components of force are $G Z \epsilon \sin \alpha$ and $G Z \epsilon \cos \alpha$.

The condition for dynamic stability is given by (48) ; i.e.,

$$
G Z \epsilon \sin \alpha>k^{\prime} T \epsilon+E_{H} \epsilon .
$$

Provided this condition is satisfied, the direction of the resultant of all the forces acting on the shaft lies behind the line of centers as shown in figure 18 . Thus the orbit of the center of the shaft should be a spiral of decreasing amplitude as shown in figure 3. 


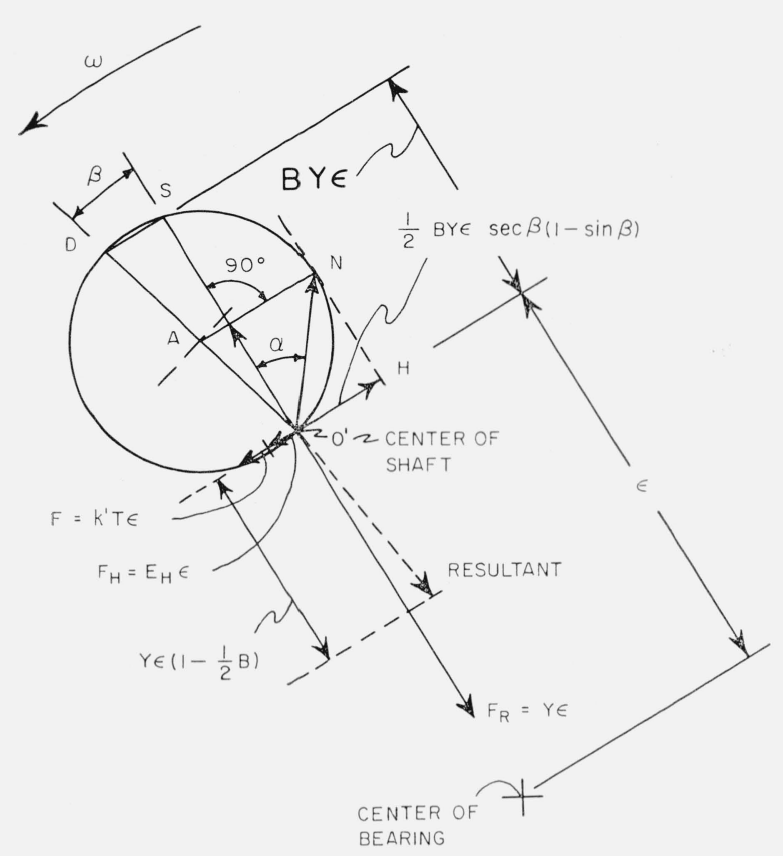

Figure 21. Vector diagram of forces illustrating design procedure.

Consequently, the bearing should be stable. This was proved experimentally in the bearings of the miniature turbine which is described by Birmingham, Sixsmith, Wilson [1962]

The design procedure and the relationship between the various forces may be illustrated with the aid of the geometrical construction shown in figure 21 . The angle $\beta$ is preferably chosen to be less than $20^{\circ}$, assuming zero whirl velocity, and the diameter of the injector holes is calculated according to (29) to satisfy this condition.

In figure 21 we have:

$$
\begin{gathered}
O^{\prime} S=B Y \epsilon \\
O^{\prime} D=B Y \epsilon \sec \beta \\
A N=1 / 2 B Y \epsilon \sec \beta \\
O^{\prime} H=A N-O^{\prime} A \sin \beta \\
=1 / 2 B Y \epsilon \sec \beta(1-\sin \beta) .
\end{gathered}
$$

Thus the stabilizing force $O^{\prime} H$ may be expressed directly in terms of the centering force $Y_{\epsilon}$. The condition for dynamic stability may now be expressed as

$$
1 / 2 B Y \epsilon \sec \beta(1-\sin \beta)>k^{\prime} T \epsilon+E_{H} \epsilon .
$$

In a trial design the number of pads and the radial clearance are adjusted until the above stability condition is satisfied. Then the diameter of the leak orifices and the volume of the stabilizing cavities may be calculated as follows.
The values of $Y_{1}$ and $Y_{2}$ according to (57) are given more simply by the equation

$$
Y_{1}=Y_{2}=Y\left(1-\frac{1}{2} B\right)
$$

and then the values of the whirl angular velocities may be calculated with the aid of (59) and (60). The diameter of the leak orifices may be calculated according to (53). Then the values of the stabilizing cavities may be calculated according to (64) and (65). This completes the design.

In the theory outlined above, it is assumed that the leakage along the shaft from the stabilizing cavities is negligible. Such leakage introduces a phase shift which tends to promote instability. Conversely, a leakage along the shaft and into the stabilizing cavities tends to promote stability. Consequently, it is desirable that the pressure at the ends of the bearing should be equal to or greater than the static pressure in the stabilizing cavities.

\section{Conclusion}

It is possible and quite practical to provide stable, externally pressurized gas-lubricated bearings for high-speed shaft operation. The stabilizing cavities employed in this design are a definite asset to a bearing system of this type and have been shown by actual tests to suppress shaft whirl inherent in externally pressurized bearings.

The bearing described here is adaptable to small shafts (one inch diameter and smaller) which are lightly loaded and require speeds well beyond the allowable operating range of rolling contact bearings. For miniature applications, the size of the bearing and shaft system is limited only by practical fabrication techniques.

This type of bearing is particularly advantageous for miniature turbine expanders used in cryogenic refrigerator systems, where the process gas can be used as the bearing gas medium. In this arrangement, the problem of contaminating the process gas from the bearing lubricant is eliminated. It also eliminates a mechanical seal between the bearings and the process expansion system.

A prototype turbine expander, incorporating externally pressurized gas-lubricated bearings, was fabricated and tested with helium gas as the operating medium. The bearings were designed using the criterion described in this manuscript. The tests showed that the bearings were reliable and performed quite satisfactorily. No detectable shaft whirl was encountered up to the design speed of the turbine shaft.

\section{Summary of Nomenclature}

$A_{1}=$ Area of pad-in. ${ }^{2}$

$A_{2}=$ Effective area of shaft surface facing cavity - in. ${ }^{2}$

$B=$ Constant defined in eq (50) 
$C=$ Capacity of cavity

$D=$ Diameter of pad-in.

$E_{H}=$ Tangential "spring" modulus-lb/in.

$F=$ Horizontal force on blading - $\mathrm{lb}$

$F_{A}=$ Static centering force- $\mathrm{lb}$

$F_{X}=$ Force due to gas pressure on shaft-lb

$F_{H}=$ Tangential force $-\mathrm{lb}$

$F_{R}=$ Radial restoring force $-\mathrm{lb}$

$F_{0}=$ Steady force $-\mathrm{lb}$

$F^{\prime}=$ Alternating vector of force- $-\mathrm{b}$

$F_{S}=$ Vector sum of forces due to $N$ cavities - $\mathrm{lb}$

$G=$ Constant defined in eq (41)

$h=$ Mean tip clearance between $\phi$ and $\phi+d \phi-$ in.

$h_{1}$

= Clearances between shaft and bearing.

See figure 9 -in.

$I_{c}=$ Alternating component of current flowing in cavity - $\mathrm{lb} / \mathrm{s}$

$I_{L}=$ Current through leak- $\mathrm{lb} / \mathrm{s}$

$I=$ Flow of gas into cavity- $\mathrm{lb} / \mathrm{s}$

$I_{0}=$ Steady component of flow into cavity$\mathrm{lb} / \mathrm{s}$

$I_{j}=$ Alternating component of flow into cavity- $\mathrm{lb} / \mathrm{s}$

$I_{a}=$ Alternating flow due to shaft rotation (eq 25)

$I_{W}=$ Alternating flow due to shaft whirl

$I_{H}=$ Alternating flow due to the combined effects of shaft rotation and whirl

$I^{\prime}=$ Alternating flow into cavity

$k=$ Constant defined in eq (17)

$k_{1}=$ Correction for overhang of rotor

$k^{\prime}=$ Constant defined in eq (46)

$L=$ Length of bearing - in.

$L_{c}=$ Effective length of stabilizing cavity-in.

$L_{1}$

$L_{2}$

$\left.L_{3}\right\rangle=$ See figure 19

$L_{4}$

$L_{5}$

$m=$ Mass of shaft in plane of bearing- $\mathrm{lb}$

$N=$ Number of pads in bearing

$n=\frac{\epsilon}{\delta}=$ Eccentricity ratio

$O=$ Center of bearing

$O^{\prime}=$ Center of shaft (see fig. 1)

$O^{\prime \prime}=$ Instant center of orbit of shaft (see fig. 2)

$P=$ Pressure in cavity-psi

$P_{0}=$ Steady component of pressure in cavitypsi

$P^{\prime}=$ Alternating component of pressure in cavity

$R=$ Resistance of leak orifice- - sec/in. ${ }^{2}$

$r=$ Radius of shaft - in.

$r_{1}=$ Turbine rotor tip radius - in.

$r_{2}=$ Turbine rotor root radius - in.

$r_{m}=$ Turbine rotor mean radius - in.

$r_{g}=$ Radius of gyration of shaft about its center of gravity - in.

$T=$ Total torque acting on turbine rotor - in.-lb

$t=$ time - sec
$V=$ Volume of stabilizing cavity (see eq (19)) in. ${ }^{3}$

$V_{1}=$ Volume of stabilizing cavities in outer bearing-in. ${ }^{3}$

$V_{2}=$ Volume of stabilizing cavities in inner bearing-in. ${ }^{3}$

$v=$ Velocity of gas at edge of injector hole-fps

$v_{1}=$ Axial component of velocity of gas through rotor tip clearance- fps

$v_{2}=$ Axial component of velocity of gas through the annulus between $r_{1}$ and $r_{2}$-fps

$Y=$ Radial spring modulus- $\mathrm{lb} / \mathrm{in}$.

$Y_{1}=$ "Spring" modulus of gas film in outer bearing- $-\mathrm{lb} / \mathrm{in}$.

$Y_{2}=$ "Spring" modulus of gas film in inner bearing- $\mathrm{lb} / \mathrm{in}$.

Greek

$Z=$ Impedance of resistance capacitance network

$\alpha=$ Phase angle between complex pressure vector and eccentricity $\epsilon$

$\beta=$ Phase angle between resultant current $I^{\prime}$ and eccentricity $\epsilon$

$\gamma=$ Angle between $\epsilon$ and a diameter through the center of a pair of diametrically opposed pads

$\delta=$ Radial clearance -in.

$\epsilon=$ Shaft eccentricity - in.

$\theta=$ See figure 2

$\mu=$ Viscosity of gas-lb-sec/in. ${ }^{2}$

$\rho=$ Density of gas in cavity $-\mathrm{lb} / \mathrm{ft}^{3}$

$\rho_{1}=$ Density of gas at edge of injector hole$\mathrm{lb} / \mathrm{ft}^{3}$

$\rho_{2}=$ Density of gas in clearance space between shaft and bearing- $\mathrm{lb} / \mathrm{ft}^{3}$

$\phi=$ See figure 8

$\omega=$ Angular velocity of whirl-radians/s

$\omega_{1}=$ Angular velocity of whirl in outer bearingradians $/ \mathrm{s}$

$\omega_{2}=$ Angular velocity of whirl in inner bearingradians/s

$\omega_{s}=$ Angular speed of shaft-radians $/ \mathrm{s}$

\section{References}

F. T. Barwell, (1956). Lubrication of bearings, p. 168. Butterworths Scientific Publications, London, England.

B. W. Birmingham, H. Sixsmith, and W. A. Wilson, (1962).

The application of gas-lubricated bearings to a miniature helium expansion turbine, Advan. Cryog. Eng., Vol., , p. 30, K. D. Timmerhaus (ed.), Plenum Press, Inc. New York, N.Y.

G. F. Boeker, B. Sternlicht, (1956). Investigation of Translatory Whirl in Vertical Machines, Trans. ASME, $88,1$.

G. W. K. Ford, D. M. Harris, and D. Pantall, (1957), Principles and applications of hydrodynamic-type gas bearings, Instit. Mech. Eng., 171, 2.

H. Sixsmith, (April 28, 1959a). Bearings for rotating shafts which are lubricated by gas, U.S. Patent No. 2,884,282, British Patent No. 797,528.

H. Sixsmith, (1959b). The theory and design of a gaslubricated bearing of high stability, Proceedings of First International Symposium on Gas-Lubricated Bearings, ACR-49, Office of Naval Research, Washington, D.C.

H. Sixsmith, W. A. Wilson, and B. W. Birmingham, (1961). Load-carrying capacity of gas-lubricated bearings with inherent orifice compensation using nitrogen and helium gas, NBS Tech. Note 115.

Paper $68 \mathrm{C} 2-156$ 